\title{
Infrared Laser Dissociation of Single Megadalton Polymer Ions in a Gated Electrostatic lon Trap: the Added Value of Statistical Analysis of Individual Events +
}

\author{
Mohammad A. Halim, ${ }^{1}$ Christian Clavier,${ }^{1}$ Xavier Dagany, ${ }^{1}$ Michel Kerleroux,${ }^{1}$ Philippe Dugourd, ${ }^{1}$ \\ Robert C. Dunbar ${ }^{2}$ and Rodolphe Antoine ${ }^{*}, 1$
}

\begin{abstract}
In this study, we report the unimolecular dissociation mechanism of megadalton $\mathrm{SO}_{3}$-containing poly(2-acrylamido-2methyl-1-propanesulfonic acid) (PAMPS) polymer cations and anions with the aid of infrared multiphoton dissociation coupled to charge detection ion trap mass spectrometry. A gated electrostatic ion trap ("Benner trap") is used to store and detect single gaseous polymer ions generated by positive and negative polarity in an electrospray ionization source. The trapped ions are then fragmented due to the sequential absorption of multiple infrared photons produced from a continuous-wave $\mathrm{CO}_{2}$ laser. Several fragmentation pathways having distinct signatures are observed. Highly charged parent ions characteristically adopt a distinctive "stair-case" pattern (assigned to the "fission" process) whereas low charge species take on a "funnel like" shape (assigned to the "evaporation" process). Also, the log-log plot of the dissociation rate constants as a function of laser intensity between PAMPS positive and negative ions is significantly different.
\end{abstract}

\section{Introduction}

Much research currently focuses on understanding the mechanism of unimolecular reactions, and relies to a great extent on mass spectrometry for large chemical and biological systems. The pioneering studies of Dunbar et al. ${ }^{1,2}$ showed that continuous-wave $\mathrm{CO}_{2}$ laser dissociation can drive a reaction and be compared with a black-body source, ${ }^{3,4}$ a phenomenon that has been exploited for the quantitative characterization of unimolecular dissociation processes. ${ }^{5-7}$ Low energy IR photons can thus promote vibrationally induced excitation, which has long been utilized to explore the dissociation mechanism of proteins and oligonucleotides in the kilodalton range, formed by electrospray ionization (ESI) coupled with Fourier transform ion cyclotron resonance mass spectrometry (FT-ICR MS). ${ }^{5-7}$ However, such studies have not been employed for megadalton species. Indeed, the mass analysis and manipulation of multiply charged ions in the megadalton range, formed by ESI, require the development of alternative detection methods in MS.

Charge-detection Mass Spectrometry (CD-MS) is a single ion measurement technique ${ }^{8,9}$ in which the mass-to-charge ratio $(\mathrm{m} / \mathrm{z})$ and charge $(z)$ of each ion can be detected individually. ${ }^{10}$ Since this technique is not only associated with the measurement of $m / z$, it can be applied to weighing large chemical and biological systems of megadalton or higher species. $^{8}, 11$ Since the first coupling of this concept with electrospray ionization (ESI) by Benner and co-workers, ${ }^{10}$ the

${ }^{1}$ Institut Lumière Matière, UMR 5306, Université Claude Bernard Lyon 1, CNRS, F69622 Lyon, France.

${ }^{2}$ Department of Chemistry, Case Western Reserve University, Cleveland, Ohio 44106, USA

tDedication: This article is dedicated to the memory of Professor Robert C. Dunbar at Case Western Reserve University.

Electronic Supplementary Information (ESI) available: [Experimental details and simulated trajectory and time of flight vs electric potential diagram of a positively charged ion. Logarithm of ion count versus trapping time for PAMPS ions without laser irradiation. Optimized structures of trimer cations and anions. SIMION simulation of the time of flight vs $\mathrm{m} / \mathrm{z}$ of high and low fragments generated from dissociation of a parent ion. Stair-case and funnel type decay. IR spectra of monomer and trimer cations and anions. Mass and MS/MS spectra of monomer AMPS cations and anions. ]. See DOI: 10.1039/x0xx00000x application of the CD-MS technique has expanded significantly to include weighing megadalton species such as DNA, ${ }^{10}, 12$ synthetic polymers, ${ }^{13}, 14$ block copolymer micelles, ${ }^{15}, 16$ vesicles, ${ }^{17}$ nanoparticles, ${ }^{18,}{ }^{19}$ fibrils $^{20}$ and viruses. ${ }^{20-29}$ In 1997, W. Henry Benner proposed an extension of the CD-MS technique by combining the charge-detection device with an electrostatic ion trap ("Benner" trap). ${ }^{30}$ Thanks to the trapping capability of this MS device, individual megadalton (masses over $10^{6} \mathrm{Da}$ ) plasmid DNA ions were detected and measured with high accuracies. Furthermore, this technique opened the way to directly measuring the dissociation kinetics of large macro ions after activation, and to providing a direct correlation between precursor and product ions. ${ }^{31}, 32$ We recently extended the limits of IRMPD to megadalton-size DNA ions, by coupling a $\mathrm{CO}_{2}$ laser to a "Benner" Trap. ${ }^{31}$ For double and single strand DNAs, 12 the experiment revealed several fragmentation pathways having distinct signatures which cannot be addressed by investigations associated with average statistical reaction rates. In a single-ion experiment, fragmentation patterns are detected and measured one ion at a time. Hence, in contrast with conventional ensemble experiments, charge and mass distributions of fragmentation pathways are measured straightforwardly.

The unimolecular dissociation mechanism may also depend on the nature of the charge of the molecular ions. Indeed, competing decay mechanisms of large hot ions can be observed for anions and cations. Previous studies on carbon clusters such as fullerenes showed different patterns of decay mechanisms for cations and anions. ${ }^{33-35}$ For fullerene cations, the decay mechanism leads to the sequential emission of neutral $C_{2}$ fragments, whereas the decay process of fullerene anions can lose one or several of its additional electrons via delayed electron emission, also known as thermionic emission. ${ }^{36}$ Competing decay mechanisms between electron loss and fragmentation is also observed for peptide polycations and polyanions following UV irradiation. ${ }^{37,38}$

Herein, we provide insight into unimolecular gas phase ion chemistry at the single ion level, in particular its dependence on the sign of the charge, in the megadalton range. We employ infrared multiphoton photodissociation coupled to a gated 
electrostatic ion trap for studying the decay mechanism of individual $\mathrm{SO}_{3}$-containing poly(2-acrylamido-2-methyl-1propanesulfonic acid) synthetic polymers labelled PAMPS (with an average molecular weight $\sim 2 \mathrm{MDa}$ ) in positive and negative polarity ESI modes. The log-log plot of the dissociation rate constants as a function of laser intensity between PAMPS positive and negative ions is quite different. The fragmentation patterns revealed by analyzing the waveforms recorded under laser irradiation are also compared, outlining the added value of statistical analysis of individual events.

a)

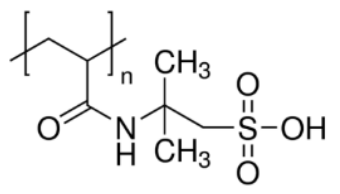

$\mathrm{Na}^{+}$

Scheme 1: a) Positive (Na+ adducts) and b) Negative (sulfonate) PAMPS ions

\section{Experimental}

Electrospray. Instrumentation and sample preparation. Megadalton mass spectrometry experiments were performed using an electrospray ionization source combined with a "Benner" trap and a $\mathrm{CO}_{2}$ laser (details are reported elsewhere). ${ }^{32}$ An electrospray ion source and vacuum interface were used to generate the ion beam. The sample solution was directly infused by ESI at a flow rate of $0.6-1.0 \mathrm{~mL} / \mathrm{h}$. For effective desolvation, nitrogen gas was used between the end cap and the transfer glass capillary. The heater temperature was set at $200^{\circ} \mathrm{C}$. We used poly(2-acrylamido-2-methyl-1propanesulfonic acid) synthetic polymer known as PAMPS, with an average molecular weight $\sim 2 \mathrm{MDa}$ (Sigma-Aldrich). Solutions were prepared in water: methanol $(50: 50, \mathrm{v} / \mathrm{v})$ at a concentration of $0.2-0.3 \mathrm{~g} / \mathrm{L}$ (agitated for $24 \mathrm{~h}$ ) and electrosprayed in both positive and negative ESI ionization modes. The sulfonate group has a high attraction for sodium ions, leading us to postulate that sodium adducts are responsible for charging in PAMPS polymers in positive ESI mode, as has been observed for the phosphate backbone in DNAs. ${ }^{10,} 39,40$ In negative ESI mode, ready deprotonation of the sulfonate backbone is likely responsible for charging in PAMPS (see Scheme 1). A spray voltage of $\sim \pm 2800 \mathrm{~V}$ was used for both ESI ionization modes. Highly charged gaseous ions were transferred through an ion optics system consisting of a hexapole and ionic lenses and focused toward the "Benner" trap. The distribution of mass for these ions recorded by electrospray coupled charge-detection mass spectrometry showed a mean mass at 2.8 MDa with a high mass tail. The later, which spreads out to $12 \mathrm{MDa}$, may have been due to the dispersion associated with the degree of polymerization for large molecules. ${ }^{7}$ The polarity of all the voltages (with the same absolute values) was reversed when negatively charged ions were studied.

Gated electrostatic ion trap: "Benner" trap. When a gaseous ion enters the trap device, it generates a circuit that enables the potentials on the entrance and exit electrodes of the ion mirrors to preset values for trapping (as described in the details in supporting information). When applying the optimal voltages on the ion mirrors, the ion trajectory oscillates between the two mirrors. The electric voltage subjected by the single trapped ion is displayed as a function of the time of flight in Fig. S1b. The ion spends about half of the TOF in the field-free region (charge detection tube) and the other half of the TOF in the ion mirrors. It is noteworthy that fragment ions are efficiently trapped when generated in the field-free region (vide infra).

When an ion is trapped in the gated electrostatic ion trap device, its charge is directly deduced from the value of the voltage intensity of the pulse generated by its entry or exit through the charge detector tube. The mass-to-charge ratio of the trapped ion is determined from the time-of-flight $\Delta \mathrm{t}$ (time delay between the positive and negative pulses that correspond to the entrance and the exit from the detector tube). The ion velocity $v_{m}$ is:

$v_{m}=\frac{L}{\Delta t} \quad$ and $\quad \frac{m}{z}=\frac{2 e V}{v_{m}^{2}-v_{g}^{2}}$

where $L$ is the length of the detector tube (i.e. $3.75 \mathrm{~cm}$ ), $m$ is the mass, $z$ is the number of charges, $V$ the electrostatic acceleration voltage. In our system, a correction is needed to take into account the initial kinetic energy imposed on the ion by the free jet expansion of the gas prior to acceleration by the electric field. $v_{g}$, is the ion velocity due to the free gas expansion. It is determined by grounding all the electrostatic lenses and timing the passage of the ion through the detector.

IRMPD in a "Benner" trap. IRMPD experiments were performed by a $25 \mathrm{~W}$ continuous wave $\mathrm{CO}_{2}$ laser with a wavelength of 10.6 $\mu \mathrm{m}$ and a diameter of $7.3 \mathrm{~mm}$ in the trap. The IR laser beam was directed by two gold-coated copper mirrors. A ZnSe window was used at the rear of the charge-detection device (CDD) chamber. The $\mathrm{CO}_{2}$ laser was operated at $5 \mathrm{kHz}$. Different laser powers were considered by varying the duty cycle from 10 to $50 \%$ to decipher the influence of the photon.

When photofragmentation occurs, the charge of the trapped ion is obtained directly from the value of the voltage intensity 
of the pulse (generated by its entry or exit through the charge detector tube) as a function of time. The mass-to-charge ratio of the trapped ion (experiencing fragmentation) can be determined only by considering the "stair-case"-type decay (vide infra).

Quantum chemistry calculations. All the calculations were performed using the Gaussian09 program. ${ }^{41}$ Model systems of AMPS monomer and trimer cations $\left(\mathrm{Na}^{+}\right)$as well as anions $\left(-\mathrm{SO}_{3}{ }^{-}\right.$ ) were optimized at the B3LYP/6-31G(d) level of theory. The vibrational frequencies of all the ions were also calculated to confirm that all the systems were within the minimum of the potential energy surface. The infrared spectra of positive and negative monomer and trimer ions were calculated at the B3LYP/6-31G(d) level of theory. A scaling factor of 0.9613 was used for IR frequencies.

\section{Results and Discussion}

\section{Fragmentation signatures at the single molecule level}

Without laser irradiation, a single ion was trapped between the ion mirrors created by the "Benner" electrostatic trap and made several hundred round trips between the ion mirrors. The image current generated by the single ion's round trip can be recorded as a function of time. Figure S2a represents the measured raw time signal for a single trapped ion generated by electrospraying a highly charged PAMPS ion (in positive mode) as it travels back and forth through the ion mirror. This highly charged single ion is trapped for $\sim 18 \mathrm{~ms}$. During this time, the ion passes $\sim 380$ times through the detector tube. In this time frame, no noticeable change was observed for the charge and mass of the single ion during the trapping time. To construct a frequency histogram of the yield of survival of the collected ions, a large set of wavelets associated with individual ions was analyzed. The yield of survival of trapped positive and negative PAMPS ions without laser irradiation is shown in Figure $\mathbf{S 2} \mathbf{b}$. Surprisingly, the yields of trapped positive and negative ions are not similar, revealing that negative ions are comparatively less stable than positive ions. The ion count remains similar up to $\sim 7.5 \mathrm{~ms}$ for both ions and then starts decreasing, in particular for negative ions. The fact that the wavelet presents no decay in the ion transient (without laser) over the time of the measurement means that the ion suddenly exits the ion trap (after $10 \mathrm{~ms}$ ). This is due to an ion that has lost kinetic energy or fragmented following collisions with the neutral molecules. Due to the size of the macromolecular ions, multiple collisions are required for ions to be lost. The background gas pressure surrounding the trap was $\sim 5 \times 10^{-6}$ mbar for this experiment. The collision cross section (ccs) for PAMPS ions is not known. However, to obtain an order of magnitude, we measured the size of PAMPS by dynamic light scattering. A hydrodynamic radius of $20 \mathrm{~nm}$ for a 2 MDa PAMPS diluted water solution was found (leading to a ccs of $1250 \mathrm{~nm}^{2}$ ). In this condition, the mean free path for single PAMPS ions is approximately $10 \mathrm{~cm}$. At this pressure, multiple ion-neutral collisions occur (PAMPS with $\mathrm{N}_{2}$ molecules) within $\sim 10 \mathrm{~ms}$ trapping time. We argue that negative PAMPS ions have more extended conformations than positive ions. Indeed, the interactions between the polymer and metal ions are assumed to involve the same kind of ion-dipole interactions as observed in cyclic polyethers, ${ }^{37}$ leading to compact folded structures. Such interactions are not present for deprotonated sites and anion structures might be more extended, as confirmed by the calculated structures of the trimer positive and negative ions (see figure S3). More extended conformations would lead to more collisions and possibly to more fragile species (vide infra). Another hypothesis stems from the slightly different values used for the ion mirror electrode voltages between the positive and negative ionization modes. Indeed, the voltage of the two L2 electrodes (which are critical for trapping ions) is $-122 \vee(+120 \mathrm{~V})$ for positively (negatively) charged ions. This difference might induce different ion trajectories in the ion trap and possibly less stable trajectories upon collisions for negatively charged ions.

During ion trapping when the $\mathrm{CO}_{2}$ laser is turned on, radical changes were observed in the oscillation shape as well as in the trapping time. A similar event reported previously for DNA macroions, ${ }^{12}$ as the waveforms recorded during laser irradiation show three distinct fragmentation patterns. In Figure 1a, no significant change in charge distribution is observed for $\sim 13 \mathrm{~ms}$. The charge amplitude then drops suddenly and the ion can no longer be detected ("sudden-loss" type fragmentation). In Figure 1b, the total charge gradually decreases before the ion is lost ( $14 \mathrm{~ms}$ ), ("funnel" type fragmentation). For the trace presented in Figure 1c, the precursor ion quickly loses a considerable amount of charge ( $\sim 45 \%$ of initial charge). After $\sim 9.5 \mathrm{~ms}$, the charge amplitude remains the same for $\sim 3 \mathrm{~ms}$ before the disappearance of the ion 


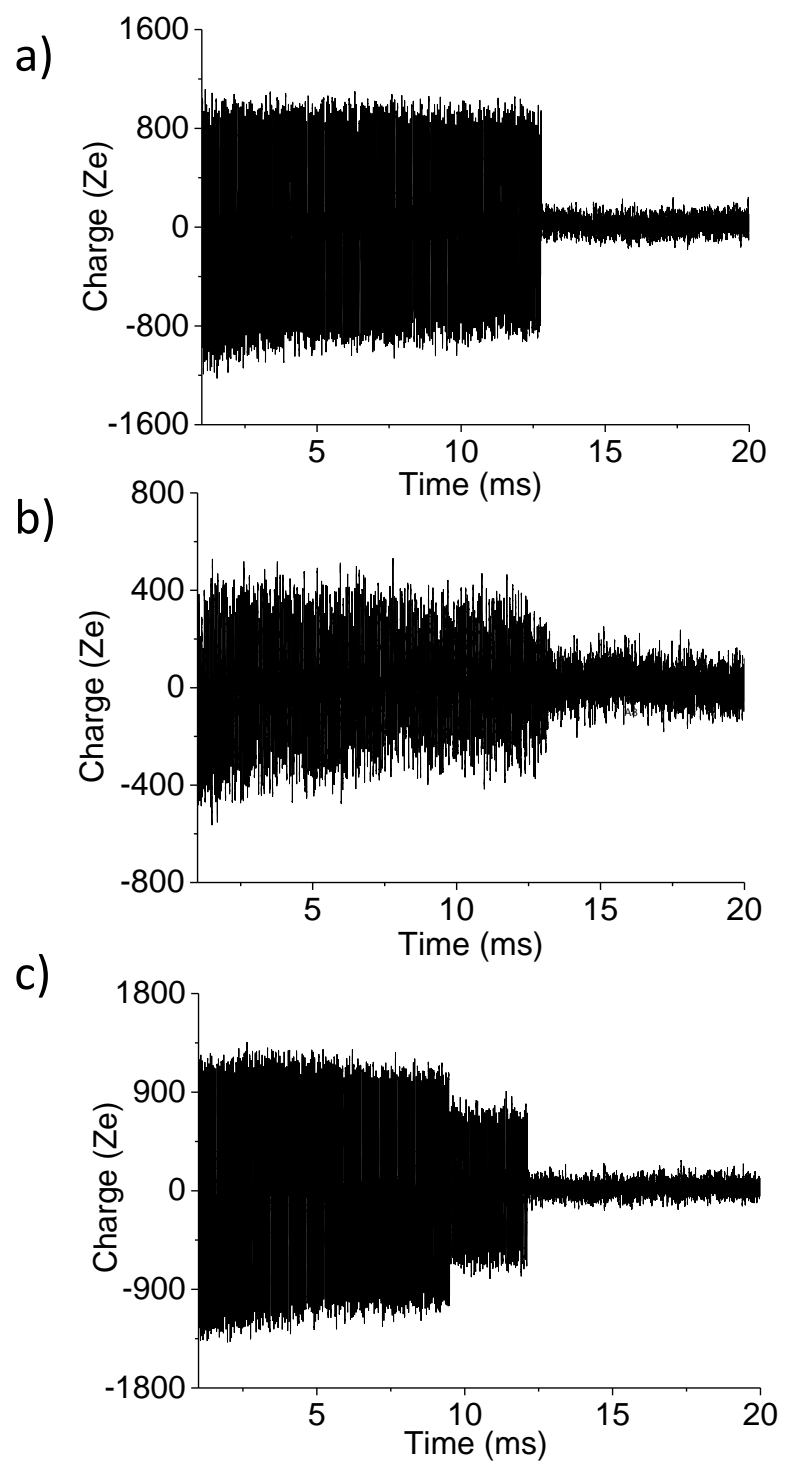

Figure 1. Ion wavelets of a) "sudden loss" b) "funnel" and c) "stair case" types of decay recorded for a PAMPS positive ion stored in the electrostatic ion trap under continuous irradiation by the $\mathrm{CO}_{2}$ laser. In the "funnel" type decay, the total charge of the ion gradually decreases before the loss of the particle.

("staircase" type of fragmentation). Note that the noise level in the pick-up signal has been improved in comparison to our previous work ${ }^{12,31,42}$ and is now $\sim 150$ e. This level corresponds to the root mean square noise level on the detector, which is typically $10 \mathrm{mV}$. This corresponds to a charge of about 150 electrons. In our analysis, we only analyzed a single fragment ion corresponding to the largest fragmentation product.

When photofragmentation occurs, and considering the "staircase"-type of decay, the $\mathrm{m} / \mathrm{z}$ ratio and the kinetic energy $\left(E_{0}\right)$ photo-fragment ions differ from the parent ion. Due to this difference, extracting accurate $\mathrm{m} / \mathrm{z}$ values of the related photofragments is difficult. The trapping stability after photofragmentation is non-trivial and requires fulfilling the condition associated with spatial and temporal focusing. ${ }^{43}$ Trajectory simulations employing SIMION 7.0 showed that fragment ions are less efficiently trapped when generated in the ion mirrors than fragments generated in the field-free region. In the ion mirrors, such fragments will usually have unstable trajectories and suddenly become lost in the trap, leading to a "sudden-loss"-type of decay. On the other hand, trajectory simulations using SIMION 7.0 indicated that fragment ions are efficiently trapped when they are generated in the field-free region (they will have the same velocity as the parent ion). If the $\mathrm{m} / \mathrm{z}$ ratio for photofragments is equal to the $\mathrm{m} / \mathrm{z}$ ratio for precursor ions, the TOF of photofragments is equal to the TOF of the parent. If the $\mathrm{m} / \mathrm{z}$ ratio for photofragment is higher (lower) than that of the parent, then the TOF of the photo fragments will be higher (lower) than that of the parent, as shown in Figure S4. A linear dependence is obtained between the $m / z$ ratio and the TOF observed for fragments. Thus, the Benner trap behaves like an MS/MS instrument, where the $m / z$ of both parent and fragments can be extracted from "staircase"-type decay. The charge and time-of-flight of parent and fragment ions can be detected separately. We collected TOFs of both parent and fragment ions (see Figure 2a) from traces belonging to "stair-case" type decay, for positively charged ions. It appears that the TOFs of parent ions are lower than the TOFs of fragment ions. This means that the largest fragmentation products generated from IR multiphoton absorption of megadalton PAMPS have higher $\mathrm{m} / \mathrm{z}$ ratios than the $m / z$ of their precursor ions. In other words, polymer ions lose more charge than mass when fragmenting. Also, from traces belonging to "stair-case"-type decay, we collected the total charges of both parent and fragment ions (see Figure $\mathbf{2 b}$ ) for positively charged ions. On average, the largest fragmentation ions hold $\sim 75 \%$ of the total parent charge. The results are similar for both cations and anions. "Stair-case"-type decay can be viewed as a kind of "asymmetric" fission of the polymer ions, while "funnel"-type decay seems to correspond to a sequential evaporation process. 

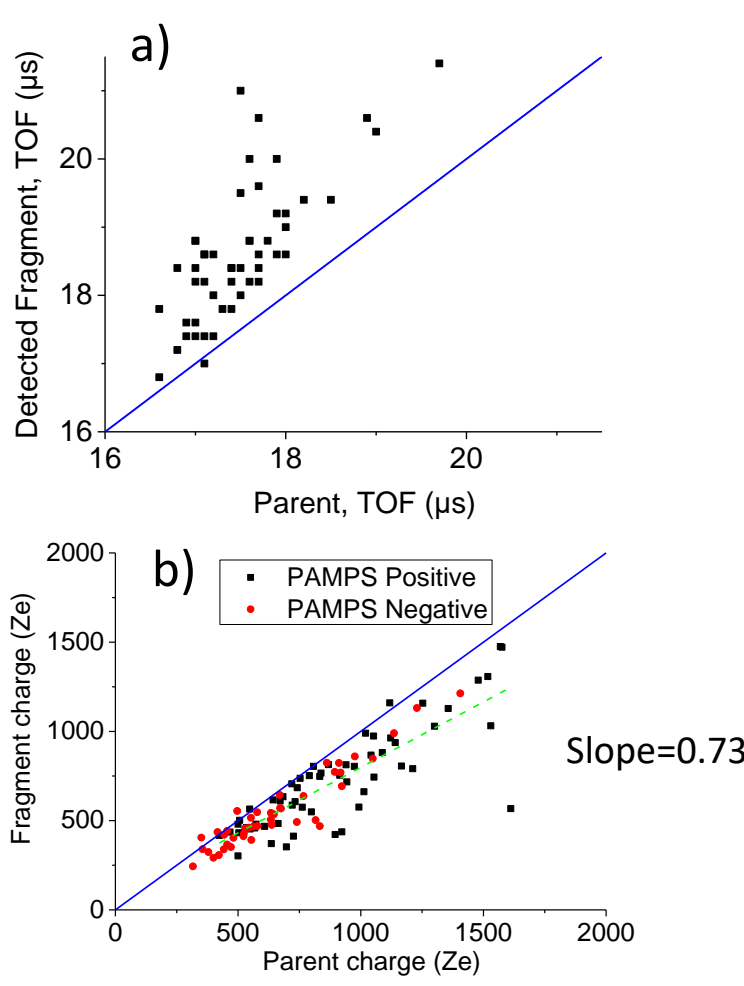

Figure 2. a) Experimental time of flight (TOF) of parent and fragment ions for positively charged ions. b) Charge distribution of parent and fragment ions in stair-case type patterns for positive and negative PAMPS ions.

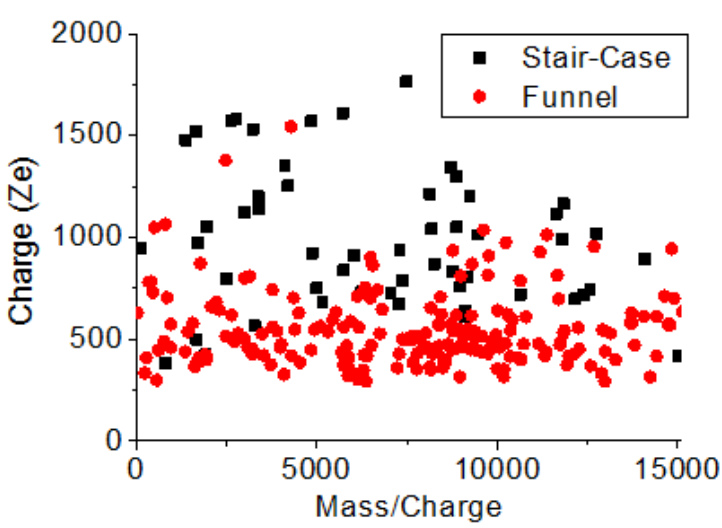

Figure 3. Charge and mass/charge plots of stair-case and funnel traces.

Figure 3 shows the distribution of "stair-case"-and "funnel"type decays in the $(m / z, z)$ landscape for positively charged
PAMPS ions. Strikingly, we observe a charge-dependent difference in propensity to follow "funnel" (sequential evaporation) vs. staircase ("asymmetric" fission) behavior. Funnel shapes are observed for lower charges in comparison to stair-case patterns, as shown in Figure 3. In particular, the staircase pattern is only observed when the charges of PAMPS ions are higher than $\sim 600$ e. For the same $\mathrm{m} / \mathrm{z}$ but a lower $\mathrm{z}$ (i.e. higher mass), the tendency is toward funnel behavior, while for the same $\mathrm{m} / \mathrm{z}$ but a higher $\mathrm{z}$ (i.e. lower mass), the tendency is toward stair-case behavior. Thus for low mass ions, the number of degrees of freedom is much smaller, which may change the branching in a statistical picture. However, a parallel can be drawn between the present finding and the fragmentation channels of large multicharged atomic clusters. ${ }^{44,45} \mathrm{~A}$ key parameter, known as fissiblity parameter, associated with the dominant fragmentation channel of a multicharged ion, can be expressed by $\mathrm{X}=\mathrm{E}_{\mathrm{c}} / 2 \mathrm{E}_{\mathrm{s}}$, in which $\mathrm{E}_{\mathrm{c}}$ and $\mathrm{E}_{\mathrm{s}}$ are the Coulomb cluster and cluster surface energy, respectively. ${ }^{44}$ At low cluster charges the evaporation of neutral monomers is the dominant "disintegration" process, whereas as the charge increases, fission processes, i.e. the emission of charged fragments, become more important. ${ }^{45}, 46$ The fissibility parameter is proportional to $q^{2} / n$, in which the net electric charge is presented by $q$ and the number of constituents of the cluster is denoted by $\mathrm{n}$. To better display the transition between the fission ("stair-case") and evaporation processes ("funnel") for charged megadalton polymer chains, using the data shown in Fig. 3, we constructed frequency count histograms for "staircase"-and "funnel"-type decays as a function of $\mathrm{q}^{2} / \mathrm{n}$ where $\mathrm{q}$ is the charge and $n$ is the number of atoms in the polymer chain (where $\mathrm{n}=\mathbf{2 7 0 0 0 0}$ is the average number of atoms in $2 \mathrm{MDa}$ PAMPS). As can be seen in Figure S5, a transition occurs between dissociation primarily by "stair-case" type to dissociation primarily by "funnel"-type at a $\mathrm{q}^{2} / \mathrm{n}$ value of $\sim 1.5$. The branching ratio of the fission ("stair-case") and evaporation ("funnel") processes for charged megadalton polymer chains appears to be determined by the value of $q^{2} / n$ for the polymer. It is noteworthy that $\mathrm{q}^{2} / \mathrm{n}$ strongly depends on the nature of the multicharged system and was measured at $\sim 0.13$ for sodium clusters ${ }^{45}, \sim 0.29$ for doubly charged gold clusters with fourteen atoms, ${ }^{47}$ and at $\sim 0.5$ for multiply charged peptide clusters. ${ }^{48}$

\section{Statistical analysis.}

A statistical analysis of the abundance of the three families of decay pattern shows that for both ions characteristic stair-case traces are relatively less frequent than other traces. The abundance of the three families is almost identical in both positive and negative modes (see Figure 4 .) with $\sim 10 \%$ staircase traces, $\sim 59 \%$ funnel-like traces and $\sim 31 \%$ sudden-loss traces (Figure 4). 

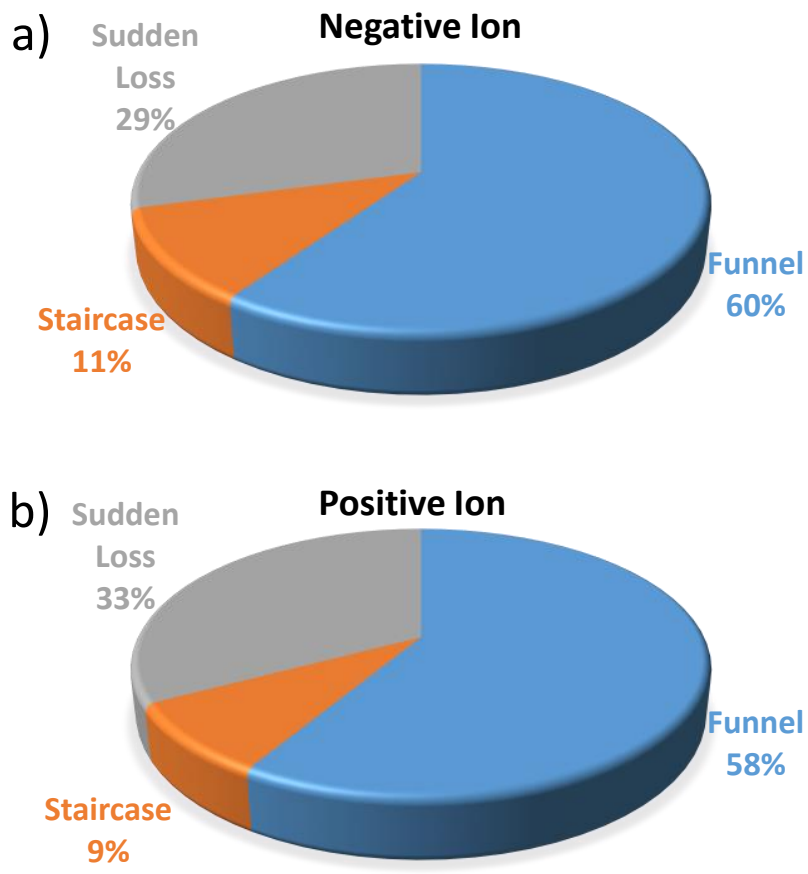

Figure 4. Distributions of the shapes of the decay wavelets for PAMPS positive and negative ions under $\mathrm{CO}_{2}$ laser irradiation. The average initial charge states for each of the three sections of the pie chart are : Stair Case $=913 e$, Funnel= $499 e$, Sudden Loss =801 e for positive mode; and Stair Case $=627$ e, Funnel= 457 e, Sudden Loss $=646$ e for negative mode.

To interpret and understand the kinetic properties associated with the dissociation of PAMPS, a large dataset of individual ion traces is required to reliably map the distribution of dissociation rates for the ions detected. Over 100 traces of individual ions were collected for each laser power. At a given time from $1 \mathrm{~ms}$ to $20 \mathrm{~ms}$, the normalized ion count is calculated by the number of traces in which the ion is still measurable at the time, and subsequent normalization is performed by the initial number of traces measured. The relative ion abundances of positive PAMPS ion as a function of irradiation time using different laser intensities from 17.9 to $29.9 \mathrm{~W} / \mathrm{cm}^{2}$ are shown in Figure $\mathbf{5}$. Similar trends are observed for negative PAMPS (data not shown). Dissociation is detected after an induction time. The dissociation kinetics follows a simple exponential decay of the precursor ions. The rate constant $k_{\mathrm{d}}$ is the inverse time constant of this exponential decay. The rate constant is obtained from the linear fit of an equation $k_{d}=-d \ln (\mathrm{Cl}) / d t$, where $\mathrm{Cl}$ is denoted as the ion count. In both positive and negative modes, the plot of $k d$ versus laser intensity in log-scale reveals a negative second derivative, as already observed for 7 MDa PEO polymers. ${ }^{42}$ At high laser power, short onset times associated with the fragmentation are obtained. These events reveal that the dissociation mechanism of the excited ions may compete with photon absorption and emission. Ion dissociation no longer occurs within the so-called rapid exchange limit (REX) ${ }^{4}$. As the laser power increases, the dissociation rate also increases and ions with slower radiative exchange rates fall outside the REX limit, while those exchanging faster remain within the REX limit until slightly higher laser powers are used. If dissociation is fast relative to activation, the thermal distribution is depleted and $k_{d}$ varies more slowly in response to high laser intensities. ${ }^{49}$ As shown in the log-scale plot of $k_{d}$ versus laser intensity, this leads to an attenuation expressing the particularly out-of-equilibrium character of dissociation under strong heating, where temperature itself is not well defined. Interestingly, this attenuation is far more pronounced for the anions than for the cations. It is not clear that the plots in Fig. 5 reach the REX limit, where they would be expected to show linear behavior, and for which the slopes could yield quantitative activation energies of the order of half an eV. However, it seems clear that the slope of the cation plot is substantially higher than that of the anion plot, indicating a substantially larger activation energy for cations than anions.
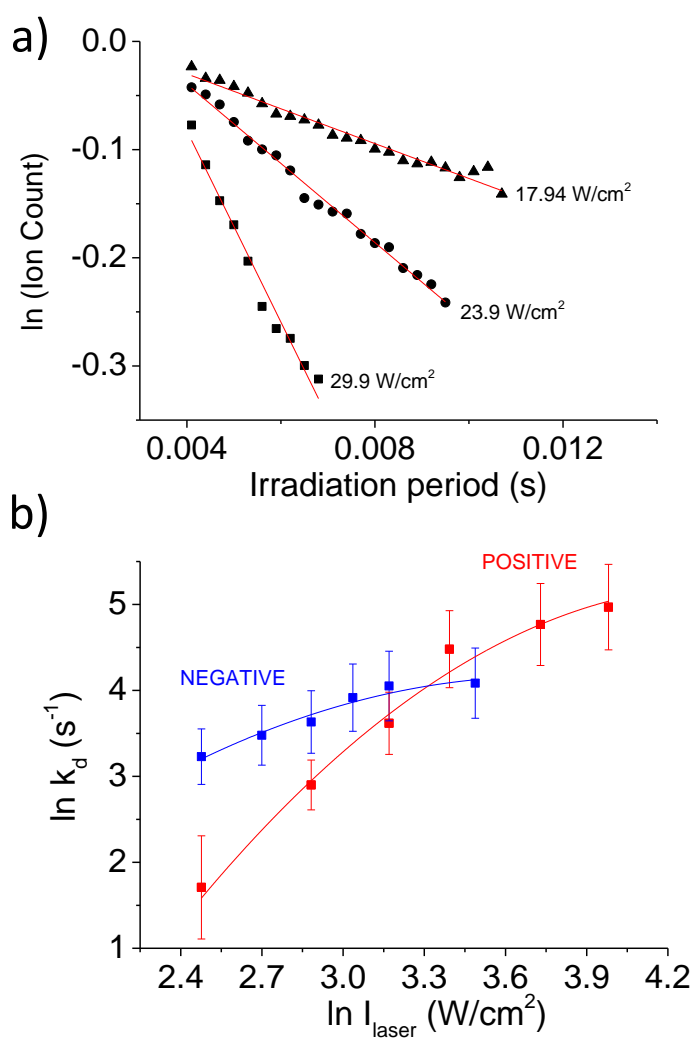

Figure 5. a) Logarithm of ion count versus time for the dissociation of PAMPS position ions under different laser irradiation powers. The plots were constructed by analyzing $\sim 300$ wavelets of individual ions at each laser power in order to construct a frequency histogram of the ion count distribution. The line corresponds to a linear fit of the data. (b) Plots of the logarithm of the first-order unimolecular dissociation rate constant, $k_{d}$, versus the logarithm of the laser intensity for both positive and negative PAMPS ions, respectively. 
Fragmentation channels: Cations versus anions.

In general, photoexcited molecular cations $\left(\mathrm{M}^{\mathrm{n}+}\right)^{*}$ can decay via (multi)ionization and (multi)electron emission, fragmentation (due to the fission or evaporation of small fragments from precursor ions), and finally by radiative transitions. The latter process is assumed to be a major process at low internal energy when fragmentation and ionization are not open. (Multi)ionization and (multi)electron emission are related to the ionization potential and the detachment energy of PAMPS polymers, respectively. The ionization potential for organic polymers ranges between 8 and $15 \mathrm{eV} .{ }^{50}$ The adiabatic electron affinity of $\mathrm{SO}_{3}$ obtained by photoelectron spectroscopy is $\sim 1.9$ eV. ${ }^{51}$ The difference observed in the activation energy of positive and negative ions might be due to different reaction channels: fragmentation for positive ions and electron loss for negative ions. However, the successive electron detachment process would lead to the observation of a gradual loss of charge from the decay traces (see Figure 1) and then a majority of "funnel"-like decay traces for negatively charged ions. The fact that the branching ratio between the 3 different families of traces (sudden-loss, funnel and stair-case) is similar for both positively and negatively charged ions precludes this hypothesis (see Figure 4).

The calculated IR spectra of positive and negative PAMPS ions revealed that the stretching frequency of the $\mathrm{S}=\mathrm{O}$ bond occurred at $955 \mathrm{~cm}^{-1}$ (correlating with the $\mathrm{CO}_{2}$ laser wavelength of $10.64 \mu \mathrm{m}=939 \mathrm{~cm}^{-1}$ ) whereas this stretching mode for positive ions is shifted to $1086 \mathrm{~cm}^{-1}$ (Figure S6). The difference observed in the evolution of $k_{d}$ versus laser intensity between positively and negatively charged ions might therefore be due to "hot spots" induced by the $\mathrm{SO}_{3}^{-}$groups, under $\mathrm{CO}_{2}$ irradiation, leading to more efficient absorption of multiple photons.

To obtain insight into reaction channels for positive and negative ions, we performed fragmentation experiments on small kilodalton polymers. We attempted to synthesize smal size polymers AMPS (in the 100-1000 Da range) via conventional free-radical polymerization. Unfortunately, due to lack of control of the polymerization process, the syntheses led to ultra-high molecular weight PAMPS. We therefore performed fragmentation experiments on AMPS monomer in positive and negative modes with a linear ion trap MS instrument according to the set-up described in ref. ${ }^{37}$

[AMPS- $\mathrm{H}+2 \mathrm{Na}]^{+}$was observed in positive ionization mode, , while [AMPS-H] $]^{-}$was observed in negative ionization mode. Fragmentation by collision activation with $\mathrm{He}$ atoms led to a major fragment corresponding to a loss of $71 \mathrm{Da}\left(\mathrm{CH}_{2}=\mathrm{CH}\right.$ $\mathrm{CONH}_{2}$ ) (Figure S7a). Fragmentation yields recorded as a function of the normalized collision energy (Figure S7c) confirmed that negatively charged AMPS fragments were more easily generated than the positively charged ones (see Figure S7b). This is in agreement with what was observed for large polymers (see Fig. S2b).

\section{Conclusions.}

Herein we coupled infrared multiphoton dissociation with charge-detection ion trap mass spectrometry to study the decay mechanism of megadalton polymer cations and anions. Our study showed that the photodissociation under $\mathrm{CO}_{2}$ laser irradiation of megadalton polymer cations and anions leads to different outcomes. Moreover, the irradiation of the trapped ions with IR photons produced three different shapes for the ion-decay profiles. Highly charged parent ions characteristically adopted a distinctive "stair-case" pattern ("fission") whereas low charge species had a "funnel like" shape ("evaporation"). A boundary was estimated at 600 e between "evaporation" and "fission" for 2 MDa polymer ions. This single-molecule experimental work outlines the benefit of studying the distribution of fragmentation pathways in large polymer ions, which cannot be inferred in ensemble experiments.

\section{Acknowledgements}

The research leading to these results received partial funding from the European Research Council in the framework of the European Union's Seventh Framework Programme (FP7/20072013 Grant Agreement No. 320659). We would like to thank Prof. Laurence Charles (Université d'Aix-Marseille - CNRS) for his useful advice.

\section{Notes and references}

1. R. C. Dunbar, J. Chem. Phys., 1991, 95, 2537-2548.

2. G. T. Uechi and R. C. Dunbar, J. Chem. Phys., 1992, 96, 8897-8905.

3. R. C. Dunbar, Mass Spectrom. Rev., 1992, 11, 309-339.

4. R. C. Dunbar and T. B. McMahon, Science, 1998, 279, 194197.

5. P. D. Schnier, J. S. Klassen, E. F. Strittmatter and E. R. Williams, J. Am. Chem. Soc., 1998, 120, 9605-9613.

6. E. Strittmatter, P. Schnier, J. Klassen and E. Williams, J. Am. Soc. Mass Spectrom., 1999, 10, 1095-1104.

7. M. A. Freitas, C. L. Hendrickson and A. G. Marshall, J. Am. Chem. Soc., 2000, 122, 7768-7775.

8. D. Z. Keifer and M. F. Jarrold, Mass Spectrom. Rev., 2017, 36, 715-733.

9. D. Z. Keifer, E. E. Pierson and M. F. Jarrold, Analyst, 2017, 142, 1654-1671.

10. S. D. Fuerstenau and W. H. Benner, Rapid Commun. Mass Spectrom., 1995, 9, 1528-1538.

11. T. Doussineau, P. Dugourd and R. Antoine, Spectroscopy Europe, 2012, 24, 16-18, 20.

12. T. Doussineau, P. Paletto, P. Dugourd and R. Antoine, J. Am. Soc. Mass Spectrom., 2015, 26, 7-13.

13. N. Ouadah, T. Doussineau, T. Hamada, P. Dugourd, C. Bordes and R. Antoine, Langmuir, 2013, 29, 14074-14081.

14. A. G. Elliott, C. C. Harper, H. W. Lin and E. R. Williams, Analyst, 2017, 142, 2760-2769. 

F. D'Agosto and B. Charleux, ACS Macro Lett., 2012, 1, 414417.

16. T. Doussineau, M. Santacreu, R. Antoine, P. Dugourd, W. Zhang, I. Chaduc, M. Lansalot, F. D'Agosto and B. Charleux, ChemPhysChem, 2013, 14, 603-609.

17. N. J. Warren, O. O. Mykhaylyk, A. J. Ryan, M. Williams, T. Doussineau, P. Dugourd, R. Antoine, G. Portale and S. P. Armes, J. Am. Chem. Soc., 2015, 137, 1929-1937.

18. T. Doussineau, A. Désert, O. Lambert, J.-C. Taveau, M Lansalot, P. Dugourd, E. Bourgeat-Lami, S. Ravaine, E. Duguet and R. Antoine, J. Phys. Chem. C, 2015, 119, 1084410849

19. B. D. Adamson, M. E. C. Miller and R. E. Continetti, EPJ Tech. Instrum., 2017, 4.

20. T. Doussineau, C. Mathevon, L. Altamura, C. Vendrely, P. Dugourd, V. Forge and R. Antoine, Angew. Chem. Int. Ed., 2016, 55, 2340-2344.

21. E. E. Pierson, D. Z. Keifer, A. Asokan and M. F. Jarrold, Anal. Chem., 2016, 88, 6718-6725.

22. E. E. Pierson, D. Z. Keifer, A. A. Kukreja, J. C. Y. Wang, A. Zlotnick and M. F. Jarrold, J. Mol. Biol., 2016, 428, 292-300.

23. D. Z. Keifer, T. Motwani, C. M. Teschke and M. F. Jarrold Rapid Commun. Mass Spectrom., 2016, 30, 1957-1962.

$24 . \quad$ D. Z. Keifer, T. Motwani, C. M. Teschke and M. F. Jarrold, J. Am. Soc. Mass Spectrom., 2016, 27, 1028-1036.

C. Chen, J. C. Y. Wang, E. E. Pierson, D. Z. Keifer, M Delaleau, L. Gallucci, C. Cazenave, M. Kann, M. F. Jarrold and A. Zlotnick, Plos Pathogens, 2016, 12.

E. E. Pierson, D. Z. Keifer, L. Selzer, L. S. Lee, N. C. Contino, J. C. Y. Wang, A. Zlotnick and M. F. Jarrold, J. Am. Chem. Soc., 2014, 136, 3536-3541.

27. A. A. Kukreja, J. C. Y. Wang, E. Pierson, D. Z. Keifer, L. Selzer, Z. Tan, B. Dragnea, M. F. Jarrold and A. Zlotnick, J. Virol., 2014, 88, 14105-14115.

28. S. D. Fuerstenau, W. H. Benner, J. J. Thomas, C. Brugidou, B. Bothner and G. Siuzdak, Angew. Chem.-Int. Edit., 2001, 40, 542-544.

29. C. A. Lutomski, N. A. Lyktey, Z. C. Zhao, E. E. Pierson, A. Zlotnick and M. F. Jarrold, J. Am. Chem. Soc., 2017, 139, 16932-16938.

30. W. H. Benner, Anal. Chem., 1997, 69, 4162-4168. Phys. Chem. Lett., 2012, 3, 2141-2145. T. Doussineau, C. Y. Bao, C. Clavier, X. Dagany, M. Kerleroux, R. Antoine and P. Dugourd, Rev. Sci. Instrum., 2011, 82.

33.

N. Walsh, A. Lassesson, F. Martinez, G. Marx and L. Schweikhard, Vacuum, 2008, 83, 761-767.

34.

35.

36.

37.

C. Lifshitz, Mass Spectrom. Rev., 1993, 12, 261-284.

C. Lifshitz, Int. J. Mass Spectrom., 2000, 198, 1-14.

C. Lifshitz, Chem. Soc. Rev., 2001, 30, 186-192.

R. Antoine and P. Dugourd, Phys. Chem. Chem. Phys., 2011, 13, 16494-16509.

38. R. Antoine, J. Lemoine and P. Dugourd, Mass Spectrom. Rev., 2014, 33, 501-522.

39. J. C. Schultz, C. A. Hack and W. H. Benner, J. Am. Soc. Mass Spectrom., 1998, 9, 305-313.

40. J. C. Schultz, C. A. Hack and W. H. Benner, Rapid Commun. Mass Spectrom., 1999, 13, 15-20.

41. M. J. Frisch, G. W. Trucks, H. B. Schlegel, G. E. Scuseria, M. A. Robb, J. R. Cheeseman, G. Scalmani, V. Barone, B. Mennucci, G. A. Petersson, H. Nakatsuji, M. Caricato, X. Li, H. P. Hratchian, A. F. Izmaylov, J. Bloino, G. Zheng, J. L.
Sonnenberg, M. Hada, M. Ehara, K. Toyota, R. Fukuda, J. Hasegawa, M. Ishida, T. Nakajima, Y. Honda, O. Kitao, H. Nakai, T. Vreven, J. A. Montgomery, J. E. Peralta, F. Ogliaro, M. Bearpark, J. J. Heyd, E. Brothers, K. N. Kudin, V. N. Staroverov, R. Kobayashi, J. Normand, K. Raghavachari, A. Rendell, J. C. Burant, S. S. Iyengar, J. Tomasi, M. Cossi, N. Rega, J. M. Millam, M. Klene, J. E. Knox, J. B. Cross, V. Bakken, C. Adamo, J. Jaramillo, R. Gomperts, R. E. Stratmann, O. Yazyev, A. J. Austin, R. Cammi, C. Pomelli, J. W. Ochterski, R. L. Martin, K. Morokuma, V. G. Zakrzewski, G. A. Voth, P. Salvador, J. J. Dannenberg, S. Dapprich, A. D. Daniels, Farkas, J. B. Foresman, J. V. Ortiz, J. Cioslowski and D. J. Fox, Wallingford CT, 2009, DOI: citeulike-articleid:9096580.

42. R. Antoine, T. Doussineau, P. Dugourd and F. Calvo, Physical Review A, 2013, 87, 013435.

43. A. L. Rockwood, J. Am. Soc. Mass Spectrom., 1999, 10, 241245.

J. Jortner, I. Last and Y. Levy, Int. J. Mass Spectrom., 2006, 249/250, 184.

45. F. Chandezon, S. Tomita, D. Cormier, P. Grubling, C. Guet, H. Lebius, A. Pesnelle and B. A. Huber, Phys. Rev. Lett., $2001,87$.

46. U. Naher, S. Bjornholm, S. Frauendorf, F. Garcias and C. Guet, Phys. Rep.-Rev. Sec. Phys. Lett., 1997, 285, 245-320.

47. W. A. Saunders, Phys. Rev. Lett., 1990, 64, 3046-3049.

48. J. C. Jurchen, D. E. Garcia and E. R. Williams, J. Am. Soc. Mass Spectrom., 2003, 14, 1373-1386.

49. R. A. Jockusch, K. Paech and E. R. Williams, J. Phys. Chem. A, 2000, 104, 3188-3196.

50. J. E. McMurry, Organic Chemistry, Enhanced Edition, Volume 1, Cengage Learning, 2009.

51. T. M. Miller, A. A. Viggiano, S. T. Arnold and J. T. Jayne, J. Chem. Phys., 1995, 102, 6021-6023. 